\title{
Correlation of Bone Marrow Aspiration and Trephine Biopsy in Various Haematological Disorders: A Study of 3 Years
}

\author{
Manoj Kumar Patro ${ }^{1}$, T Santosh ${ }^{2 *}$, Atanu Kumar Bal ${ }^{3}$, Anita Choudhury ${ }^{3}$, Jayanti Nayak ${ }^{3}$, \\ Bodhisatwa Behera $^{3}$ and Debi Prasad Mishra ${ }^{3}$ \\ 'Department of Pathology, Government Medical College \& Hospital, Balangir, Odisha, India \\ ${ }^{2}$ Department of Pathology, Pathkind Labs, Varanasi, India \\ ${ }^{3}$ Department of Pathology, MKCG Medical College \& Hospital, Berhampur, Odisha, India
}

\begin{abstract}
Background: The bone marrow examination is an essential investigation for the diagnosis and management of many disorders of the blood and bone marrow. The aspirate and trephine biopsy specimens are complementary and when both are obtained, they provide a comprehensive evaluation of the bone marrow. The present study was conducted to compare the role of trephine biopsy with bone marrow aspiration for effectively diagnosing wide spectrum of hematological diseases. Few studies have compared the relative value of aspirate with trephine biopsy.

Materials \& Method: This is a three year observational study undertaken in Dept. of Pathology, MKCG MCH, Berhampur,Odisha. A total of 370 cases presented with haematological disorders, of which only126 patients had undergone trephine biopsy and correlation was done with aspiration in these patients.

Results: Of a total 370 patients, both BMA \& BMB were performed on 126 patients (71male \& 55 female). Commonly encountered diseases were AML (17\%), IDA (11\%), ALL (9\%), others (9\%), CML (8\%), (6\%) accounted for maximum number of cases. Other conditions included TB, NPD, Metastatic Diseases such as SRBCT, NHL\& Neuroblastoma. Patients from 2 months to 80 years old were encountered in the study. BMB was diagnostic in $100 \%$ cases. In comparison BMA, a positive diagnosis was made in $80 \%(101)$ cases, suggestive in $6.3 \%(8)$ cases \& negative in 13.4\%(17) cases. BMB was superior to BMA in diagnosis of MF (2\%), ALL (4\%), Others (9\%) where BMA aspirations yielded a dry tap / diluted marrow. In the present study BMB was advantageous in diagnosis \& staging in 19\%(25) cases. Additional advantages of $\mathrm{BMB}$ noted in the present study were assessment of cellularity, detection of Abnormal localization of immature precursors,, assessment of fibrosis, nodular/diffuse/focal patterns of involvement, metastatic deposits, and granuloma could be identified in BMB.

Conclusion: The decision whether to perform a BMA alone or in combination with BMB rests on the diagnostic possibilities. In IDA, ITP \& Acute leukaemia's where cellular morphology is desired aspiration is best. BMB is superior when assessment of cellularity, detection of ALIP, assessment of fibrosis, nodular/diffuse/focal patterns of involvement, metastatic deposits, granuloma, with the use of IHC on BMB samples the accuracy in diagnosis of Lymphoma, AML/ALL, Multiple myeloma \& Metastatic Diseases can be made. Thus BMA \& BMB should always go hand in hand.
\end{abstract}

\section{Keywords: Bone Marrow Aspirate, Bone Marrow Biopsy, Myelofibrosis, Niemann Pick Diseases, Gaucher's Disease,} Metastatic Carcinoma, Small Round Blue Cell Tumor.

\section{Introduction}

Bone marrow is involved in variety of haematological and non-haematological disorders. The haematological disorders include acute leukemia, myeloproliferative neoplasm (MPN) diagnosis and staging of hematolymphoid neoplasm, nutritional deficiency diseases. On the other hand non-haematological disorders include infectious diseases infiltrating the bone marrow such as tuberculosis, parasitic infections and metastatic deposits. ${ }^{[1]}$ Although, diseases of bone marrow present with various clinical symptoms and also involve the blood but peripheral blood picture alone does not reflect the nature of disease process. Depending upon diagnosis suspected from the clinical features and peripheral blood examination, indications for bone marrow examination can be RBC disorder , WBC disorders, Storage disorders, Iron store assessment, Granulomatous conditions, Metastasis, Post chemotherapy patients, etc. Therefore, complete haematological evaluation of cases where bone marrow examination was indicated includes BMA smear and trephine BMB as they are complementary to each other providing complete cytomorphology, differential count of cells, marrow architecture with distribution of tumor cells and further IHC or flow cytometric analysis can also be done. These procedures are also valuable for follow up of patients undergoing chemotherapy, bone marrow transplantation and other forms of medical treatment. ${ }^{[2,3]}$ at MKCG Medical College, 
Berhampur, Odisha to evaluate the complementary role of both the procedures done simultaneously and to see the advantages and disadvantages of these procedures.

Aim of our study was to look for the spectrum of various haematological disorders, examine bone marrow morphology in various bone marrow disorders \& to compare the incidence of the underlying pathology and correlate the bone marrow findings with clinical findings and peripheral blood findings. To evaluate the utility and accuracy of BMA \& BMB individually and in combination.

\section{Materials and Method}

This was a prospective study conducted in the Department of Pathology, MKCG Medical College \& Hospital, Berhampur over a period of 3 years. During the period 370 bone marrow aspirations were done of which 126 cases had simultaneous bone marrow core needle biopsy. The protocol followed in each case was - [i] recording of detailed clinical history and other investigation findings, [ii] Complete blood counts (CBC) and peripheral smear study in each case followed by [iii] BMA \& BMB.

Protocol for BMA ${ }^{[3]}$ : Needle - Salah's / Klima's bone marrow aspiration needle; Site - Posterior superior iliac spine was the most common site, others being anterior superior iliac spine, upper end of tibia. Aspirated material was expressed on to multiple slides and wedge smears and crush smears were prepared and air dried. Smears were routinely stained with Leishman stain and Pearl's stain for iron. Different cytochemical stains performed as per case necessity were myeloperoxidase (MPO), Sudan black B (SBB), Periodic acid schiff's (PAS), Zeel Nelson stain for acid fast bacilli (AFB), etc.

Protocol for $\mathrm{BMB}^{[3]}$ : Needle - Jamshedi's needle; Site Posterior superior iliac spine. Procedure: After marrow was aspirated biopsy needle with trocar in position was introduced through the same skin puncture site, the outer table was punctured perpendicularly at a site close to the aspiration wound. Then the trocar was removed and the needle was directed towards the anterior superior iliac spine, with a constant firm pressure the needle was progressed with a rotatory movement. Approximately 2 to $3 \mathrm{~cm}$ of the needle has to be passed to obtain a marrow core of adequate length. Adequate core of marrow is meant by more than $2 \mathrm{~cm}$ length and at least 10 well preserved intertrabecular spaces are available for evaluation. Imprint slides were prepared. Subsequently the cores were processed for 12 hours in $10 \%$ NBF followed by decalcification in $10 \%$ EDTA solution for 24 to 48 hours. Finally the decalcified cores were routinely processed by the automatic tissue processor, embedded and $3-4 \mu \mathrm{m}$ thick sections were prepared. All were routinely stained with Hematoxylin and Eosin (H \& E) stain, Reticulin \& Pearls stain. Other stains performed were PAS, ZN stain etc.

All biopsies and aspirates were independently studied by two pathologists. The following features were recorded in each case:

\section{BMA:}

- Nature of material- Dry tap / Bloody tap / Normal marrow particles.

- Adequacy.

- Cellularity.

- Routine microscopic findings - Leishman stain, Pearl's stain.

- Other ancillary study findings - MPO, SBB, PAS, ZN stain etc.

- Diagnostic or not.

BMB:

- Quality of microscopy.

- Adequacy.

- Cellularity.

- Topographic arrangement of cells.

- Routine microscopic findings - H \& E stain, Pearl's stain.

- Other ancillary study findings - MPO, SBB, PAS, ZN stain etc.

- Diagnostic or not.

\section{Result}

A total of 370 cases were taken for evaluation, of which 126 had undergone both aspiration and biopsy. These cases were further reviewed in our study. The average length of biopsies in our study was $1.2-1.6 \mathrm{~cm}$ (Mean=1.5cm). Of the 126 cases, $71(56.3 \%)$ were male \& 55(43.7\%) female patients. There was a positive correlation between BMA and $\mathrm{BMB}$ in $101(80.2 \%)$ cases whereas in 17 cases (13.4\%) diagnosis was made only from BMB (Table 1). No definite opinion could be given either from BMA or $\mathrm{BMB}$ in 8 cases $(6.4 \%)$.

Our study showed a highest correlation rate with erythroid hyperplasia 23/23 (100\%) cases and acute myeloid leukemia 22/22 (100\%) cases (Figure 1). In those cases where a diagnosis of erythroid hyperplasia 23 cases (18.2\%) were given, depending on whether macro-normoblastic or micro-normoblastic, they were further worked up mostly for anaemia and accordingly Perl's stains for iron was done, 
biochemical parameters were taken into consideration and the impression was given. Iron stains were better appreciated in BMA rather than $\mathrm{BMB}(76 \%$ showed iron stain grade $1 \& 2$ in BMA whereas were negative in BMB).

Other cases with a good positive correlation were hematological malignancies such as Chronic myeloid leukemia $10 / 10(100 \%)$, Immune thrombocytopenic purpura showed $100 \%$ correlation $(2 / 2)$ and multiple myeloma 6/8 (75\%) cases. We also had cases of AML M6 $\&$ ALL (Figure 2). CLL (Figure 3). BMA was reported as suggestive of aplastic anaemia in 16 cases but BMB revealed hypo cellular marrow only in 9 cases where as aplastic anaemia in rest of 7 cases.

Amongst the non-hematological malignancies; NHL comprised 4 cases, metastatic to the bone marrow 3 cases, tuberculosis of bone marrow 3 cases, Niemann pick disease were 2 cases. Of the non hematological malignancies only $6(50 \%)$ cases showed positive correlation in both BMA and BMB. The 3 case of metastasis to bone marrow had primaries in breast (Figure 4), neuroblastoma \& small blue round cell tumor.

92 cases were having good cellularity on BMA \& BMB while 4 hypocellular in BMA, 5 hypocellular in BMB, while
25 cases with dry tap were excluded in the comparison. Reticulin stain for fibrosis was done in BMB samples, in which Myelofibrosis 2/2 cases (Figure 5), CML 2/10 case \& hypoplastic marrow 7/16 cases, respectively showed grade 3 fibrosis (Graph 1).

BMA yield was dry tap in 25 cases which included Non hematological malignancies (6), Aplastic anemia (6), Hypoplastic marrow (5), ALL (4) cases, Multiple myeloma and Myelofibrosis 2 each, MDS (1) respectively. (Graph 2) These cases were later diagnosed by BMB.

Additional information such a tumor cell type focal granuloma (Figure 6) \& pattern of involvement was better appreciated from BMB in metastatic cases, cellularity in case of hypoplastic marrow, plasma cell percentage \& pattern of involvement in multiple myeloma, pattern of involvement of marrow in case of CLL, fibrosis in hairy cell leukemia (Table 2). Our diagnostic accuracy in BMA with $\mathrm{BMB}$ was $86 \%$. The remaining $13 \%$ cases $\mathrm{BMB}$ was only helpful for diagnosis.

\section{Discussion}

BMA and BMB are important diagnostic procedures for diagnosis of hematological, non-hematological malignancies and other diseases. These procedures are also

Table 1: Correlation of diagnosis established on BMA \& BMB.

\begin{tabular}{|l|c|c|c|}
\hline \multirow{2}{*}{ TYPE OF CASES } & \multicolumn{2}{|c|}{ DIAGNOSIS ESTABLISHED BY } & \multirow{2}{*}{ TOTAL } \\
\cline { 2 - 3 } & BMA+BMB (CONCORDANCE) & BMB (DISCORDANCE) & 23 \\
\hline IDA+MA & $23(100 \%)$ & 0 & 22 \\
\hline AML & $22(100 \%)$ & 0 & 10 \\
\hline CML & $10(100 \%)$ & 0 & 6 \\
\hline CLL & $6(100 \%)$ & 0 & 2 \\
\hline ITP & $2(100 \%)$ & 0 & 2 \\
\hline HYPERSPLEENISM & $2(100 \%)$ & 0 & 2 \\
\hline SA & $2(100 \%)$ & 0 & 1 \\
\hline HPS & $1(100 \%)$ & 0 & 1 \\
\hline HES & $1(100 \%)$ & 0 & 1 \\
\hline HCL & 0 & $1(100 \%)$ & 2 \\
\hline MF & 0 & $2(100 \%)$ & 6 \\
\hline MDS & $5(83.3 \%$ & $2(16.7 \%)$ & 8 \\
\hline MM & $6(75.0 \%)$ & $4(33.0 \%)$ & 12 \\
\hline ALL & $8(66.6 \%)$ & $6(50.0 \%)$ & 12 \\
\hline METASTATIC & $6(50.0 \%)$ & $9(57.0 \%)$ & 16 \\
\hline HYPOPLASTIC & $7(43.0 \%)$ & $\mathbf{2 5 ( 1 9 . 8 \% )}$ & $\mathbf{1 2 6 ( 1 0 0 \% )}$ \\
\hline TOTAL & $\mathbf{1 0 1 ( 8 0 . 1 \% )}$ & & \\
\hline
\end{tabular}


Table 2: Additional findings obtained from BMB.

\begin{tabular}{|c|c|c|c|}
\hline Type Of Cases & No. & Additional Findings & No. Of Cases With Add. Findings \\
\hline AML & 22 & Fibrosis \& cellularity assessment & 5 \\
\hline IDA & 14 & Nil & 0 \\
\hline ALL & 12 & Cellularity & 4 \\
\hline Metastatic & 12 & $\begin{array}{c}\text { Tumor cell type , focal granulomas, } \\
\text { pattern of involvement }\end{array}$ & 12 \\
\hline CML & 10 & Fibrosis & 10 \\
\hline Hypo plastic & 16 & Cellularity (hot spot) & 12 \\
\hline MA & 8 & -- & 0 \\
\hline MM & 8 & Plasma cell $\%$, Pattern of involvement & 3 \\
\hline CLL & 6 & Pattern of involvement & 6 \\
\hline MDS & 6 & Blast \% & 4 \\
\hline ITP & 2 & Nil & 0 \\
\hline MF & 2 & Fibrosis & 2 \\
\hline Hyperspleenism & 2 & Nil & 0 \\
\hline SA & 2 & Iron stores & 0 \\
\hline HES & 1 & Eosinophil \% & 0 \\
\hline $\mathrm{HCL}$ & 1 & Fibrosis \& cell character & 0 \\
\hline HPS & 1 & Nil & 0 \\
\hline
\end{tabular}

Table 3: Advantages and disadvantages of BMA \& BMB

\begin{tabular}{|c|c|}
\hline \multicolumn{2}{|c|}{ ADVANTAGES AND DISADVANTAGES OF BMA \& BMB } \\
\hline BMA & BMB \\
\hline \multicolumn{2}{|c|}{ ADVANTAGES: } \\
\hline $\begin{array}{c}\text { Comparatively easy to perform with less discomfort to the } \\
\text { patient. }\end{array}$ & $\begin{array}{l}\text { Features like cellularity and topographic relationship of } \\
\text { different marrow elements is better appreciated. }\end{array}$ \\
\hline Cellular morphology is better appreciated. & $\begin{array}{c}\text { Multiple sections will be available for various ancillary stains } \\
\text { and their comparative evaluation. }\end{array}$ \\
\hline Suitable for Cytochemical stains. & Suitable for ancillary studies like Immunohistochemistry. \\
\hline $\begin{array}{c}\text { Suitable for other ancillary studies like Flow cytometry, } \\
\text { cytogenetics, Culture, etc. }\end{array}$ & -- \\
\hline \multicolumn{2}{|c|}{ DISADVANTAGES: } \\
\hline Cellularity cannot be properly assessed. & More discomfort to the patient. \\
\hline Dry tap can occur. & Time consuming. \\
\hline Possibility of missing focal diseases. & Cellular morphology cannot be assessed. \\
\hline
\end{tabular}

\begin{tabular}{|c|c|c|c|c|c|c|}
\hline \multicolumn{7}{|c|}{$\begin{array}{r}18 \\
16 \\
14 \\
12 \\
10 \\
8 \\
6 \\
4 \\
2\end{array}-$} \\
\hline & MF & AML & $\mathrm{CML}$ & ALL & ET & Hypoplastic \\
\hline Gr. 0 & 0 & 18 & 5 & 12 & 1 & 0 \\
\hline Gr. 1 & 0 & 3 & 3 & 0 & 0 & 3 \\
\hline Gr. 2 & 0 & 1 & 0 & 0 & 0 & 7 \\
\hline Gr. 3 & 2 & 0 & 1 & 0 & 0 & 6 \\
\hline Gr.4 & 0 & 0 & 0 & 0 & 0 & 0 \\
\hline
\end{tabular}

Graph 1: Grading of fibrosis 


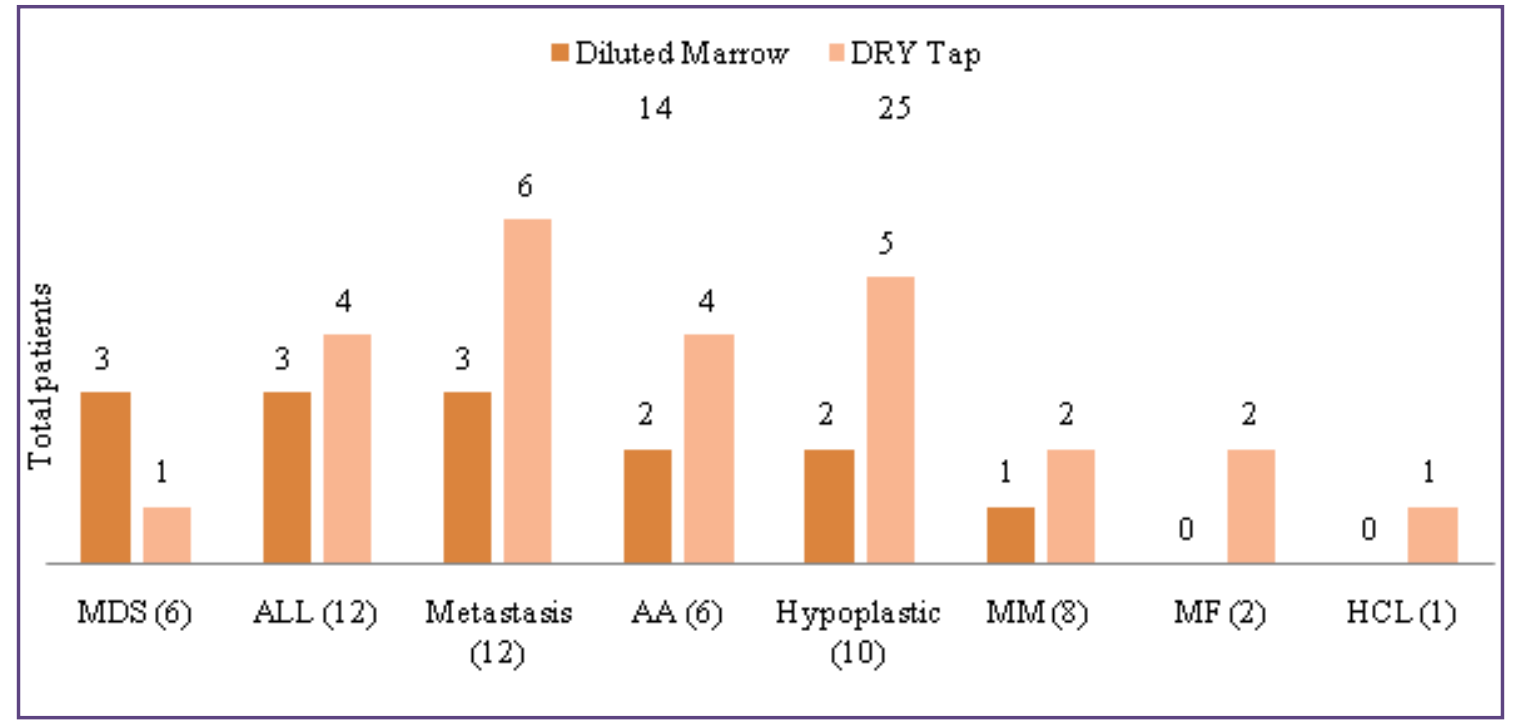

Graph 2: Causes of failure in BMA

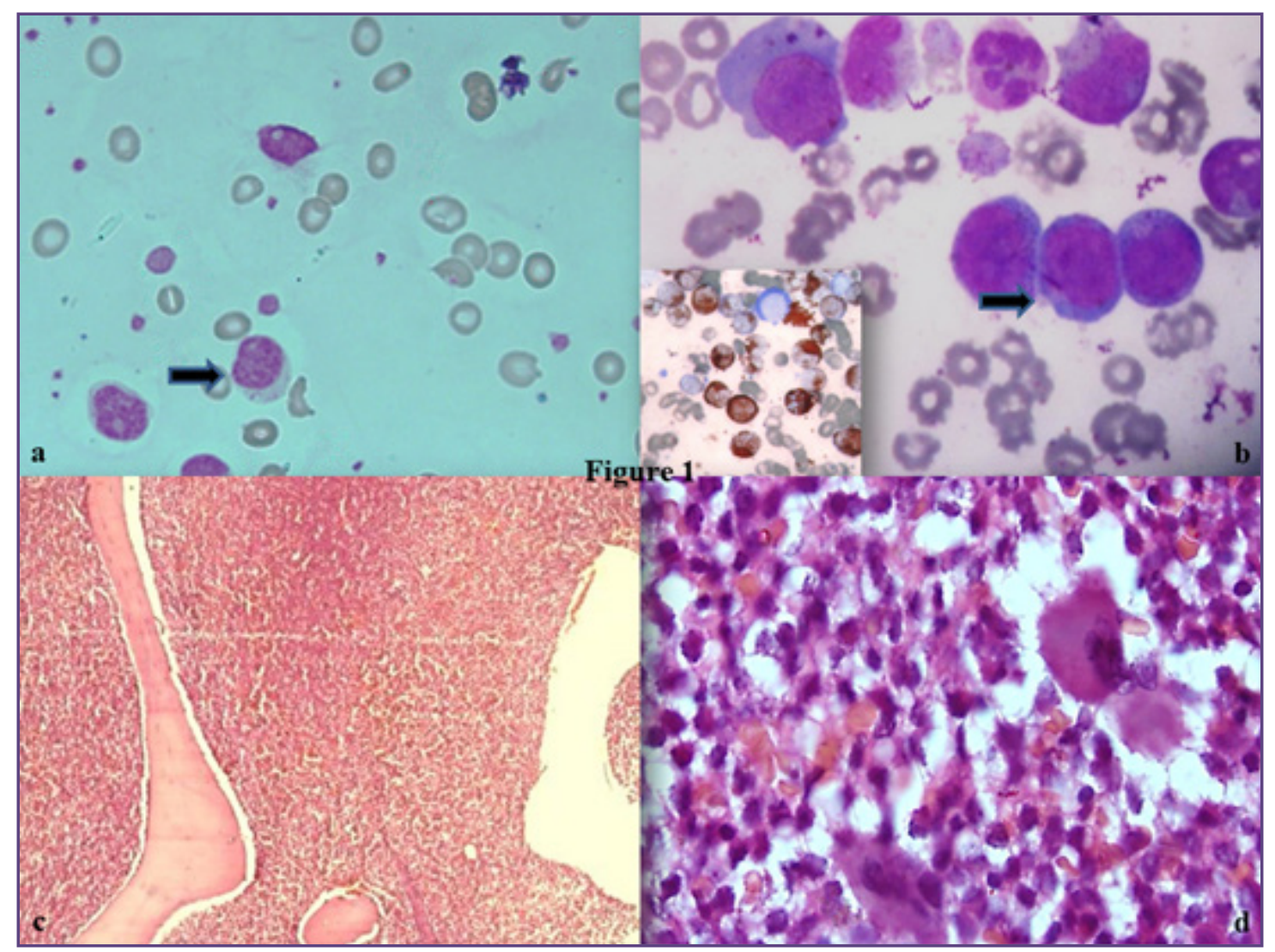

Fig. 1a: AML M3- PS showing blast with auer rods (arrow); Figure 1b: BMA with blast showing auer rods and MP0 positive; Figure 1c,d: BMB cellular with myeloblast and megakaryocyte. (MGG,x40; MP0, x40; H\&E, x40). 


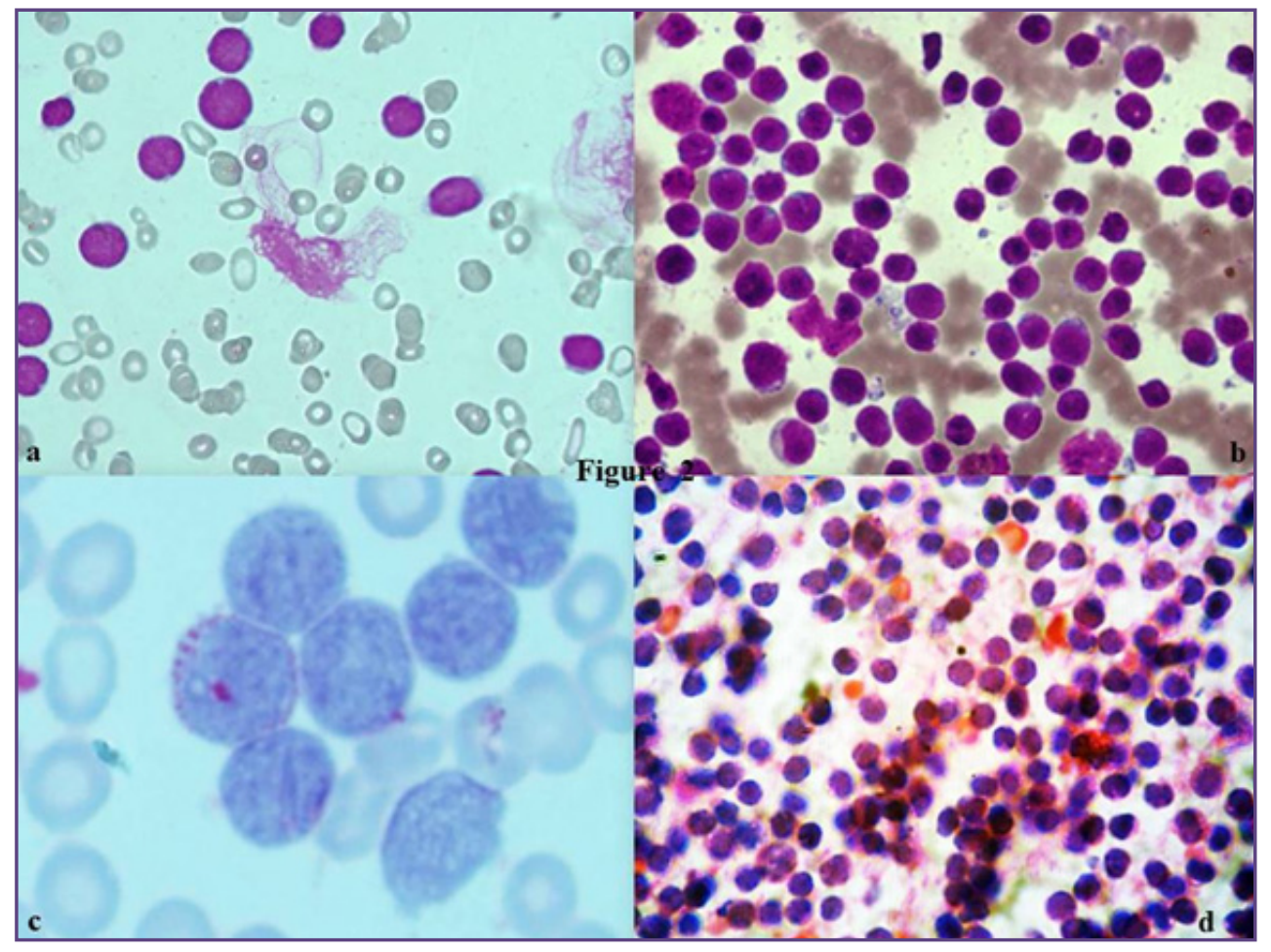

Fig. 2a: ALL L1- PS with lymphoblast and smudge cell; Figure 2b: BMA with lymphoblast which are positive for PAS satin (Figure 2c); Figure 32d: BMB with lymphoblast replacing marrow space. (MGG,x40; PAS, x40; H\&E, x40).

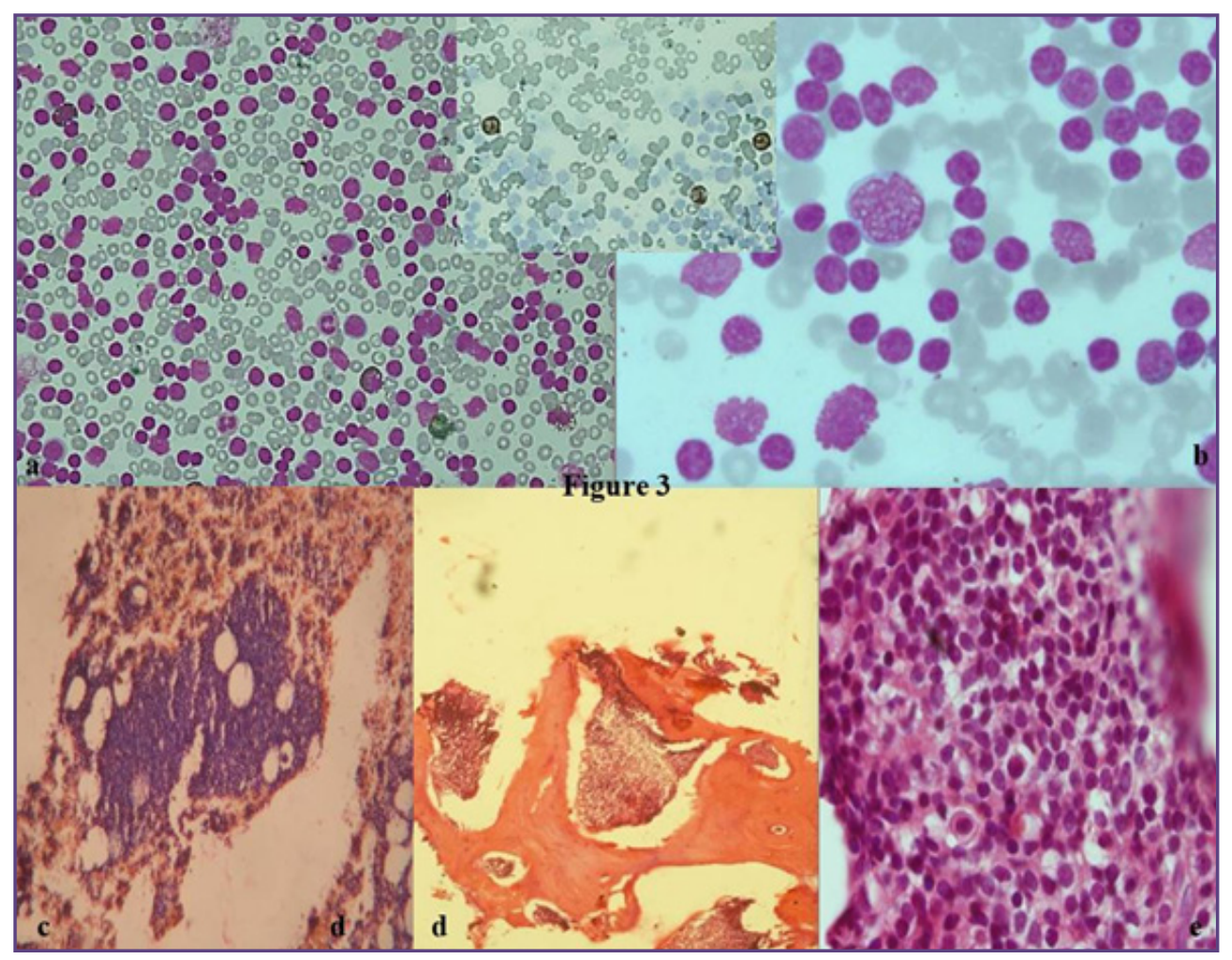

Fig. 3a,b: CLL- PS \& BMA cellular with blast negative for MPO(inset); Figure 3c:cell block with mature lymphoid cells; Figure 3d,e: BMB with mixed pattern of lymphoid infiltrate. (MGG,x40; H\&E, x40). 


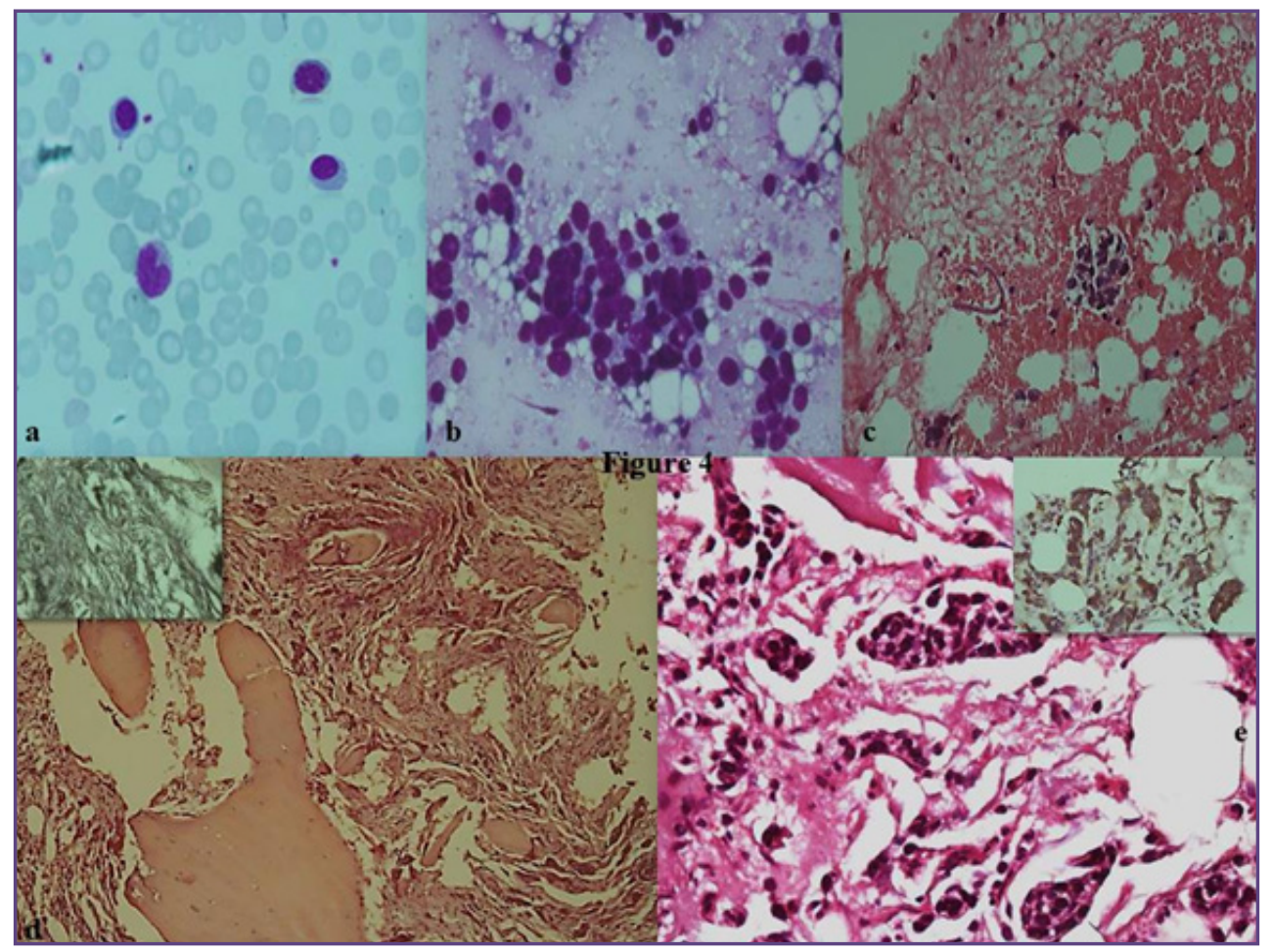

Fig. 4a: Metastasis of Breast carcinoma to BM- PS showed leucoerythroblastic features; Figure 4b: BMA showed clusters of tumor cells; Figure 4c: Cell block with tumor cell and microfilaria; Figure 4d,e: BMB with tumor cell and increased fibrosis and IHC positive for Her-2/neu. (MGG,x40; H\&E, Reticulin,IHC x40)

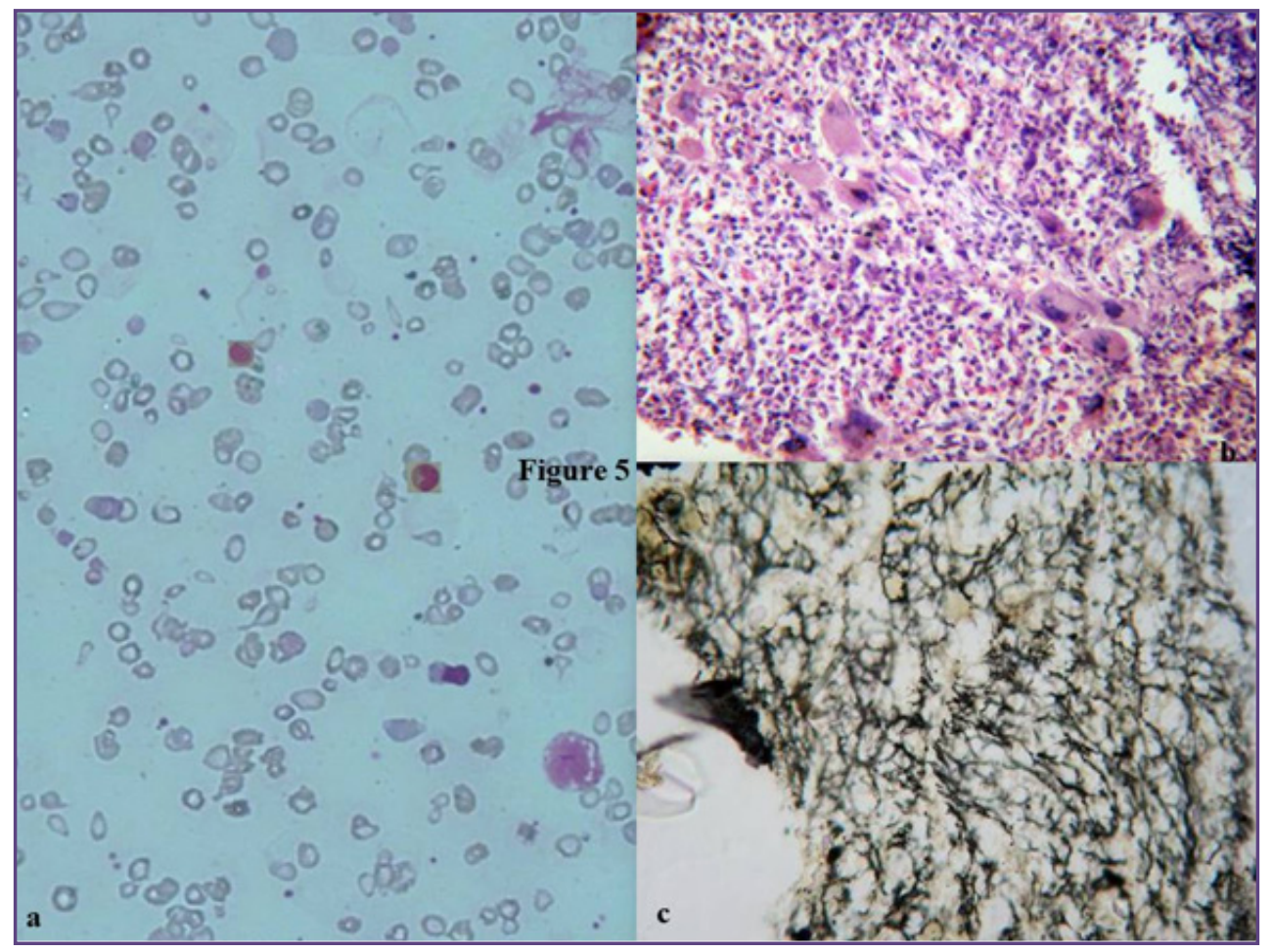

Fig. 5a: Myelofibrosis- PS with leucoerythroblastic features; Figure 5b: BMB showing atypical megakaryocytes with increased fibrosis (Figure 5c,). (MGG, x40; H\&E, Reticulin, x40). 


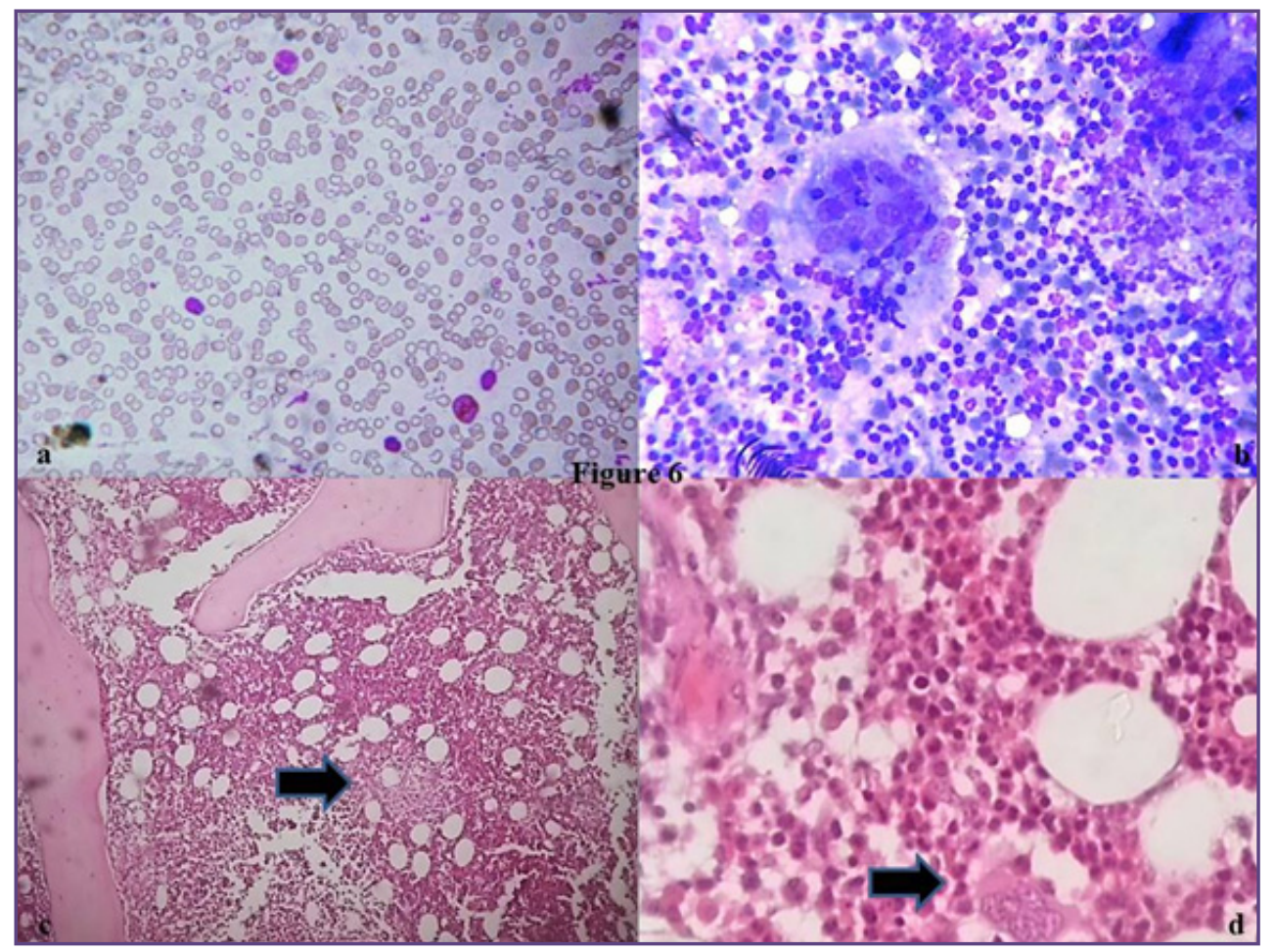

Fig. 6a: TB involving BM- PS showing features of pancytopenia; Figure 6b: BMA showed epithelioid granulomas and necrosis; Figure 6c,: BMB with epithelioid granuloma and Langhen's giant cells (Figure 6d). (MGG, x40; H\&E, x40).

valuable for follow up of patients undergoing chemotherapy, bone marrow transplantation and other forms of medical treatment. ${ }^{[2,4]}$ It is a well-known fact that bone marrow aspiration and bone marrow biopsy complement each other. Now-a-days both specimens are routinely obtained at the same time and usually same site. ${ }^{[4]}$ In our study, we did a comparative evaluation of all such BMA and BMB, to see the complementary role of both the procedures, to study the advantages and disadvantages of both the procedures done simultaneously (Table 3).Erythroid hyperplasia was the most common diagnosis in BMA accounting for a total of 23 cases $(18.25 \%)$ in our study. Similar incidence (14\%) was shown in a study by Khodke et al. ${ }^{[5]}$ Jha et al in their study showed an incidence of $19.6 \%{ }^{[6]}$ These cases showing erythroid hyperplasia with either macronormoblastic or micronormoblastic proliferation were further worked up. Perl's stain was done and the biochemical parameters were taken into consideration. The diagnosis was given either as iron deficiency anaemia or megaloblastic anaemia. But iron stained sections of BMB showed differences in iron content from that of BMA smears Iron deficiency anemia cases with Perl's stain showed better results with aspirate (60\% grade 1) compared to biopsy (13.3\% grade 1$)$. These observations were nearly similar to the findings seen in a study conducted by $\mathrm{Ch}$ Toi $\mathrm{P}$ et al. ${ }^{[2]}$ Stuart-Smith SE et al., have also shown in a study that aspirate smears reflect bone marrow iron stores more reliably than acid decalcified trephine biopsy sections. ${ }^{[7]} 75 \%$ (6/8 cases) of multiple myeloma showed a positive correlation in BMA and BMB. Although it was not difficult to diagnose multiple myeloma in BMA alone, where the aspirate was good, there were cases where the plasma cells were scattered. In such cases BMB complemented the BMA, as it helps to identify compact masses of plasma cells with no stroma as observed by Sabarhwal et al. ${ }^{[8]}$ Our study correlated with the study done by Charles et al. ${ }^{[9]}$ where they detected myeloma in trephine biopsies and all simultaneous bone marrow aspirates.

30 cases $(23.8 \%)$ of acute leukemia; acute lymphoblastic leukemia and acute myeloid leukemia, were diagnosed in BMA in our study compared to 4 cases which were diagnosed on BMB. The predominant reason for not diagnosing acute leukemia on BMA in our case was dry tap either due to marrow fibrosis or tightly packed marrow by leukemic cells.

Other haematological malignancy in which we observed good concordance rates were CML 10 cases, 6 cases each of CLL and Multiple myeloma, 5 cases of MDS. Both bone marrow aspiration and trephine biopsy were complementary in all the other cases of malignancies diagnosed in our study. While aspiration smears were observed to be most effective 
for studying cellular morphology, biopsy on the other hand, was helpful in assessing marrow cellularity, pattern of involvement by leukemic cells. Our findings are comparable to the study by James et al who observed that combined procedures of aspiration and biopsy gave a higher yield and are essential in patients with leukemia and lymphoma. ${ }^{[10]}$ Hence, it is important that both the aspirated and biopsy material should be examined together, since the two methods are often complementary. ${ }^{[1]}$ Also, trephine biopsy permits an accurate assessment of extent of infiltration and gives information of prognostic importance. ${ }^{[12]}$

Our study had 2 cases, where BMA yielded dry tap was diagnosed as myelofibrosis on trephine biopsy. Trephine biopsy was also superior to BMA in diagnosing 6 cases of hypoplastic/ aplastic anemia similar to study by Gupta $\mathrm{N}$ et al. ${ }^{[13]}$ Sabharwal et a ${ }^{[8]}$ included 7 cases $(23.3 \%)$ of myelofibrosis which were diagnosed on trephine biopsy sections. Other cases of dry tap in our study were ALL, metastasis, aplastic anemia, hypoplastic marrow myeloma and HCL. In a study done by Humphries of 87 cases of dry tap on marrow aspiration, obtained trephine biopsies which showed significant pathology. ${ }^{[14]}$ Hence, the finding of a dry tap should never be dismissed as being due to faulty technique and always needs a bone marrow biopsy.

One case of tuberculosis was diagnosed by BMA, while 2 cases detected by biopsy. This could mainly be due to focal involvement of the marrow by granulomas which is very difficult to be detected on aspirate smears. Ch Toi P et al., have mentioned that $80 \%$ cases of granulomatous lesions were diagnosed by BMB alone. ${ }^{[2]}$

There were four cases of NHL in the present study where BMB biopsy rendered information which cannot be determined from aspiration such as spatial distribution and extent of infiltrates, overall cellularity and fibrosis. This also implies that trephine biopsy may be more useful in post chemotherapy patients to assess the residual tumour cell burden and degree of chemotherapy response. ${ }^{[15]}$

Microfilaria was identified in one case with $100 \%$ correlation between BMA and BMB. Similar finding was seen in a study done by Santra et al. ${ }^{[16]}$

Hence, it was observed from the above discussion that bone marrow evaluation is an important cum effective tool in diagnosing and evaluating hematological and non-hematological disorders. ${ }^{[1,17]}$ Complete evaluation of bone marrow samples includes a brief patient history, hematological profile, marrow aspirate smears and biopsy sections. ${ }^{[18]} \mathrm{A}$ correlation of bone marrow aspiration and biopsy showed that both the procedure were complementary to each other. The BMA generally provides an excellent cytomorphological details which enables hematopathologist in recognising the abnormal hematopoietic cells or the non-native cells in case of non-hematological disorders. Whereas, a bone marrow trephine biopsy demonstrates the topographic arrangement of hematopoietic cells within the marrow framework and hence gives a more representative view of the cellularity of the marrow and allows infiltration to be recognized clearly. ${ }^{[19]}$ BMB examination has definite edge over BMA in the detection of minimal residual diseases, staging of lymphoma and for the diagnosis of acute leukemia in relapse cases which are otherwise clinically silent. ${ }^{[1]}$

\section{Conclusion}

The present study showed that BMA and BMB are easy, rapid, cost-effective and more or less are of equal value in various hematological and non-hematological disorders of bone marrow. The study concludes that bone marrow aspiration cytology and trephine biopsy complement each other and should be performed simultaneously for complete bone marrow workup and evaluation. Though cellular morphology is better understood in marrow aspirates and is equally effective to biopsy in diagnosing anemia and leukemia, it is the histopathological study of trephine biopsy of bone marrow that gives well preserved marrow architecture with its all cellular, stromal components, architectural patterns, grading of fibrosis, pattern of infiltration with lymphomas and granulomatous conditions. So, trephine biopsy becomes mandatory in the diagnosis of aplastic anemia, myelofibrosis and granulomatous involvement yielding dry aspirate on bone marrow aspiration. The evaluation of BMA \& BMB comprises the complete work up for proper bone marrow interpretation and to reach final diagnosis.

Ethics Approval and Consent to Participate: All procedures performed in the study involved human participants were in accordance with the ethical standards of the institutional ethics committee.

Consent for Publication: Written consent was obtained from individual participants included in the study.

Availability of Data and Materials: All the data regarding the findings are available within the manuscript.

Authors' Contributions: TS carried out concepts \& design, literature search, participated in clinical study. MK carried out data acquisition, data analysis \& manuscript preparation will stand as guarantor also. $\mathrm{AB}$ carried out concepts \& design, literature search. SB participated in clinical study \& manuscript review. BB carried out literature search \& data acquisition. DM participated in clinical study \& manuscript review. All the authors have 
read \& approved the final manuscript.

\section{References}

1. Manjit K, Amrit R, Shivali K, Arun P. Diagnostic Value of Bone Marrow Aspiration and Biopsy in Routine Hematology Practice. Journal of Clinical and Diagnostic Research 2014;8(8):13-16.

2. Ch Toi P, Varghese G'Boy R, Rai R. Comparative evaluation of simultaneous bone marrow aspiration and bone marrow biopsy. An institutional experience. Indian J Hematol Blood Transfus. 2010;26(2):41-44.

3. Lee SH, Erber WN, Porwit A, Tomonga M, et al. ICSH guidelines for the standardization of bone marrow specimens and reports. Int. Jnl. Lab. Hem. 2008;30:349-64.

4. Islam A. Bone marrow aspiration prior to bone marrow core biopsy using the same bone marrow biopsy needle. A good or bad practice. J Clin Pathol. 2007;60:212-15.

5. Knodke K, Marwah S, Buxi G, Yadav RB, Chaturvedi NK. Bone marrow examination in cases of Pancytopenia. $J$ Academy Clin Med 2001;2:55-59.

6. Jha A, Sayami G, Adhikari RC, Panta D, Jha R. Bone marrow examination in a case of pancytopenia. J Nepal Med Assoc 2008;47:12-17.

7. Stuart_SE, Hughes DA, Bain BJ. Are routine iron stains on bone marrow trephine biopsy specimens necessary? J Clin Pathol. 2005;58(3):269-72.

8. Sabharwal BD, Malhotra VV, Aruna SS, Grewal RR. Comparative evaluation of bone marrow aspirate particle smears, imprints and biopsy sections. J Postgrad Med 1990;36:194-98.

9. Charles KS, Winfield DA, Angel C, Goepel J. Audit of bone marrow aspirates and trephine biopsies in multiple myeloma-a single centre study. Clin Lab Hematol 2004; 26:403-06.

10. James D, Bearden, Gary A, Ratkin,Charles AC. Comparison of the diagnostic value of bone marrow biopsy and bone marrow aspiration in neoplastic disease. J Clin Pathol 1974;27:738- 40.

11. Sudha HM, Krishnappa R, Rishi B. Correlation of Bone Marrow Aspirate, Biopsies and Touch Imprint Findings in Pancytopenia. J Hematol 2013;2(1):8-13

12. Kim YS, Ford RJ, Faber JA. B-cell chronic lymphocytic leukemia/small lymphocytic lymphoma involving bone marrow with an interfolicular pattern. Am J Clin Pathol. 2000;114(1):41-46.

13. Gupta V, Tripathi S, Tilak V, Bhatia BD. A study of clinicohaematological profiles of pancytopenia in children. Trop Doct. 2008;38(4):241-43.

14. Humphries JE. Dry tap bone marrow aspiration: clinical significance. Am J Hematol. 1990;35(4):247-50.

15. Goyal S, Singh UR, Rusia U. Comparative Evaluation of Bone Marrow Aspirate with Trephine Biopsy in Hematological Disorders and Determination of Optimum Trephine Length in Lymphoma Infiltration. Mediterr J Hematol Infect Dis. 2014;6(1):e2014002. doi: 10.4084/ MJHID.2014.002. eCollection 2014.

16. Santra G, Das B K. A cross-sectional study of the clinical profile and aetiological spectrum of pancytopenia in a tertiary care centre. Singapore Med J 2010;51:806-12. PMid:21103817

17. Parajuli S, Tuladhar A. Correlation of bone marrow aspiration and biopsy findings in diagnosing hematological disorders - a study of 89 cases. Journal of Pathology of Nepal 2014;4:534 -38.

18. Smita C, Harish C. Comparison of bone marrow aspirate cytology, touch imprint cytology and trephine biopsy for bone marrow evaluation. Hematology Reports 2011;3(22):65-68.

19. Surbhi G, Usha RS, Usha R. Comparative Evaluation of Bone Marrow Aspirate with Trephine Biopsy in Hematological Disorders and Determination of Optimum Trephine Length in Lymphoma Infiltration. Mediterr J Hematol Infect Dis 2014, 6(1): e2014002, DOI: 10.4084/MJHID.2014.002.

*Corresponding author:

Dr. T Santosh, Lab Head, Pathologist Lab-Operations, Pathkind Labs, Varanasi, India

Phone: +91 8895495670,09584873660

Email: born_vss@yahoo.co.in

Financial or other Competing Interests: None. 